\title{
The Tonkin weakfish, Cynoscion similis (Sciaenidae, Perciformes), an endemic species of the Amazonas- Orinoco Plume
}

\author{
Rodrigo Antunes CAIRES ${ }^{1}$, Wagner. C. R. dos SANTOS ${ }^{2,3}$, Leonardo MACHADO ${ }^{4}$, Claudio OLIVEIRA5, \\ Najila N. C. D. CERQUEIRA 5 , Matheus Marcos ROTUNDO ${ }^{6}$, Cintia OLIVEIRA7, \\ Alexandre P. MARCENIUK ${ }^{2,4 *}$ (1) \\ ' Universidade de São Paulo, Museu de Zoologia (MZUSP), São Paulo, SP, Brazil \\ 2 Instituto Chico Mendes de Conservação da Biodiversidade (ICMBio), Centro de Pesquisa e Gestão de Recursos Pesqueiros do Litoral Norte (CEPNOR), Belém, PA, Brasil \\ 3 Universidade Federal Rural da Amazônia (UFRA), Belém, PA, Brasil \\ ${ }^{4}$ Instituto Federal de Educação, Ciência e Tecnologia de Santa Catarina, Centro de Referência em Navegação e Pesca Marítima, Itajaí, SC, Brasil \\ Universidade Estadual Paulista Júlio de Mesquita Filho (UNESP), Instituto de Biociências, Departamento de Morfologia, Botucatu, SP, Brasil \\ ${ }^{6}$ Universidade Santa Cecília, Acervo Zoológico, Santos, SP, Brasil \\ Museu Paraense Emílio Goeldi (MPEG), Belém, PA, Brazil \\ * Corresponding author: a_marceniuk@hotmail.com; (1D https://orcid.org/0000-0003-4286-0482
}

\section{ABSTRACT}

Despite its importance in biogeographical, ecological, and commercial terms, the fish fauna of the northern Brazilian coast is still poorly known, representing the least sampled portion of the Brazilian Exclusive Economic Zone. We collected Tonkin weakfish, Cynoscion similis specimens during extensive surveys of the northern Brazilian coast and concluded that C. similis is common in this region. While the species had not previously been reported for the northern Brazilian state of Pará, it may have been recorded in studies of industrial fisheries, being identified only as Cynoscion sp. or by the common name pescada negra. This reinforces the need for the reliable taxonomical identification of species, to guarantee the collection of accurate data on ecology and fisheries, and ultimately, support the development of effective conservation strategies. Here we provide additional morphological and molecular data to distinguish Cynoscion similis from the closely related Cynoscion jamaicensis, and other congeners.

KEYWORDS: Brazilian north coast, Cynoscion jamaicensis, industrial fisheries, DNA barcoding, reliable taxonomical identification

\section{Pescada negra, Cynoscion similis (Sciaenidae Perciformes), uma espécie endêmica da pluma Amazonas - Orinoco}

\section{RESUMO}

Apesar de sua importância em termos biogeográficos, ecológicos e comerciais, a ictiofauna da costa norte do Brasil ainda é pouco conhecida, representando a porção menos amostrada da Zona Econômica Exclusiva Brasileira. Durante extensivo esforço de inventário na costa norte do Brasil, nós obtivemos uma série de espécimes de Cynoscion similis e concluímos que a espécie é comum nesta regiấo. Embora a espécie não tenha sido relatada anteriormente para o estado do Pará, ela pode ter sido registrada em estudos sobre a pesca industrial, sendo identificada apenas como Cynoscion sp. ou pelo nome comum pescada negra. Isso reforça a necessidade de identificação taxonômica confiável de espécies, com objetivo de garantir dados precisos de ecologia e pesca, e apoiar o desenvolvimento de estratégias efetivas de conservação. Aqui, nós fornecemos novos dados morfológicos e moleculares importantes para a diferenciação de Cynoscion similis e C. jamaicensis, a espécie mais próxima morfologicamente, assim como de outros congêneres.

PALAVRAS-CHAVE: costa norte do Brasil, Cynoscion jamaicensis, pesca industrial, DNA barcoding, identificação taxonômica 


\section{INTRODUCTION}

The Tonkin weakfish or pescada negra, Cynoscion similis Randall \& Cervigón, 1968, was described from specimens collected on the coast of Venezuela, Suriname, and French Guiana. Since its description, however, the species has only rarely been mentioned in the literature (Chao 1978, 2002, 2003; Uyeno et al. 1983; Cervigón 1992, 1993; Aguillera 1998; Casatti and Menezes 2003). Cynoscion similis is very similar to the Jamaican weakfish, Cynoscion jamaicensis (Vaillant and Bocourt 1883), being distinguished from the latter species by the presence of a sheath of 1-2 scales on the base of the 2 nd dorsal fin and the anal fin, which, in $C$. jamaicensis, is covered with small scales over at least half of its total extension (Chao 2003).

In a review of the classification of the sciaenids of the western North Atlantic, Chao (1978) noted that Cynoscion similis is found from "Venezuela to Brazil", although its occurrence on the Brazilian coast is known only from records from the state of Amapá (http://www.fishnet2.net). This position was perpetuated in subsequent publications, as in the paper of Uyeno et al. (1983) on the fish species trawled off Suriname and French Guyana, and in the list of Brazilian marine fishes (Casatti and Menezes 2003). In the IUCN Red List account for $C$. similis, however, it is stated that "There (are) no known records of this species from Brazil (F. LucenaFrédou, pers. comm. 2009)" (Chao and Aguillera 2015)

We conducted extensive surveys on the northern Brazilian coast, and, during these surveys, we collected a number of Cynoscion similis specimens. In this context, the present study reports the occurrence of Cynoscion similis on the northern coast of Brazil, and provides a new morphological diagnosis of the species, together with an analysis of its DNA barcode.

\section{MATERIAL AND METHODS}

\section{Material examined}

Material examined in the present study is held by the following Brazilian institutions: the zoological collection (Acervo Zoológico) of Universidade Santa Cecília (AZUSC) in Santos, São Paulo State, Brazil; Museu Paraense Emílio Goeldi (MPEG) in Belém, Pará State, Brazil; and the Zoology Museum (Museu de Zoologia) of Universidade de São Paulo (MZUSP) in São Paulo, São Paulo State, Brazil.

Cynoscion similis 22 specimens 213-309 mm SL: MPEG 35794 (1, 286 mm SL), Amapá, 0344’N, 50¹2’W, 72 m; MPEG 35807 (1, 299 mm SL), Amapá, 0339’ N, 50¹0’W, 66 m; MZUSP 68853, (3), Amapá, 259’N, 49²2'W; MPEG 35605 (2, 241-269 mm SL), Amapá, 02¹9’N, 48³6’W; MPEG 3568 (1, 269 mm SL), Amapá, 2¹7’N, 48³3’W; MPEG 35612 (1, 264 mm SL), Amapá, 02¹7’ N, 48³3'W; MPEG 35696 (2, 241-269 mm SL), Amapá, 02¹9’N, 4836'W; MPEG 35590 (1, 255 mm SL), Amapá, 02¹4’N,
48²5'W; MPEG 35679 (1, 300 mm SL), Amapá, 0209’N, $48^{\circ} 27^{\prime} \mathrm{W}, 47$ m; MPEG 35743 (2, 158-184 mm SL), Amapá, $02^{\circ} 07^{\prime} \mathrm{N}, 48^{\circ} 26^{\prime} \mathrm{W}$; MPEG 35752 (2, 256-263 mm SL), Amapá, 0207'N, 48²6'W; MPEG 35691, (1, 303-309 mm SL), Amapá, $02^{\circ} 07^{\prime} \mathrm{N}, 48^{\circ} 26^{\prime} \mathrm{W}, 44$ m; MPEG 35636 (1, 224 mm SL), Amapá, 0204'N, 48²6'W, 42 m; MPEG 35509 (2, 232-240 mm SL), Amapá, 01²5’N, 4844'W; MPEG 35105 (2, 272-284 mm SL), Amapá, $01^{\circ} 15^{\prime} \mathrm{N}, 48^{\circ} 00^{\prime} \mathrm{W}, 46 \mathrm{~m}$; MPEG 35042 (2, 213-223 mm Sl), Pará, 0003'S, 47³1'W, 38 m; AZUSC 4650 (1, 240 mm SL), Pará, 0054’' 4, 46³7'W.

Cynoscion_jamaicensis, 29 specimens 142-285 mm SL: MPEG 35743 (2, 158-184 mm SL), Amapá, 0207’N, $48^{\circ} 26^{\prime} \mathrm{W}$; MPEG 35588 (1, 152 mm SL), Amapá, 02²14'N, $48^{\circ} 25^{\prime} \mathrm{W}$; MPEG 35587 (1, $171 \mathrm{~mm}$ SL), Amapá, 0206'N, 483’ W; MPEG 35508 (3, 146-184 mm SL), Amapá, $01^{\circ} 25^{\prime} \mathrm{N}, 48^{\circ} 04^{\prime} \mathrm{W}$; MPEG 35678 (9, 152-166 mm SL), Amapá, 01²5'N, 4804'W; MPEG 35683 (1, 160 mm SL), Amapá, 0209'N $48^{\circ} 27^{\prime} \mathrm{W}$; MPEG 35689 (1, 179 mm SL), Amapá, 0207'N, 48²6'W; AZUSC 1764 (4, 164-285 mm SL), São Paulo, Santos; AZUSC 2778 (3, 142-157 mm SL), São Paulo, Santos; AZUSC 4719 (4 173-187 mm SL), São Paulo, Santos.

\section{Morphological analysis}

Measurements were taken either with a ruler and recorded to the nearest millimeter, or with dial calipers and recorded to the nearest $0.1 \mathrm{~mm}$. The following measurements were obtained through orthogonal projections using a ruler: Caudal - peduncle length $(\mathrm{CPl})$, from the posterior basal margin of the last anal-fin ray to the end of the hypural plate; Distance from snout to anal fin (SAd), from the tip of the snout to the anterior basal portion of the first anal-fin spine; Distance from snout to dorsal fin (SDd), from the tip of the snout to the anterior basal portion of the first dorsal-fin spine; Distance from the tip of the snout to the pectoral fin (SPd), from the tip of the snout to the anterior basal portion of the first pectoralfin ray; Head length $(\mathrm{Hl})$, from the tip of the snout to the end of the operculum, including its membranous portion; Snout length $(\mathrm{Sl})$, from the tip of the snout to the anterior margin of the orbit; Standard length (SL), from the tip of the snout to the end of the hypural plate. The remaining measurements were taken point-to-point: Anal fin height (Ah), from the insertion to the end of the longest spine; Anal-fin base length $(\mathrm{Abl})$, from the anterior basal margin of the first spine to the posterior basal margin of the last ray; Body depth $(\mathrm{Bd})$, the greatest vertical distance between the anterior basal margin of the first dorsal-fin ray to the anterior basal margin of the first anal-fin ray; Body width $(\mathrm{Bw})$, greatest body width at the pectoral girdle; Caudal peduncle height $(\mathrm{CPh})$, least depth of the caudal peduncle; Dorsal fin height (Dh), from the basal insertion to the tip of the longest spine; Dorsal-fin base length $(\mathrm{Dbl})$, from the anterior basal margin of the first dorsal-fin spine to the posterior basal margin of the last dorsal-fin ray; 
Head depth (Hd), the greatest vertical height of the head at the posterior margin of the operculum; Maxillary height (Mh), greatest vertical height of the maxilla at the mouth angle; Maxillary length $(\mathrm{Ml})$, greatest distance between the anterior and posterior margins of the maxilla; Mouth width (Mw), external distance between mouth angles; Orbital diameter $(\mathrm{Od})$, greatest orbital width; Interorbital distance (IOd), the greatest distance between the upper margins of the orbit; Postorbital length (POl), the distance from the posterior margin of the orbit to the end of the membranous posterior portion of the operculum; Pectoral fin height (Ph), from the basal insertion to the tip of the longest ray. Counts were taken of the elements of the dorsal, anal and pectoral fins, as well as the number of gill rakers on the first gill arches, the number of scales with pores on the lateral line, and the number of scales above and below the lateral line, at the level of the dorsal fin. Whenever possible, the sex of the specimens was determined through the examination of the morphology of the gonads under magnification.

Principal Component Analysis (PCA) was used to analyze the log-transformed measurements in a matrix of co-variance. The values that were constant were excluded from the PCA, so the measurements included were $\mathrm{Abl}, \mathrm{Bd}, \mathrm{CPh}, \mathrm{CPl}, \mathrm{Dh}$, $\mathrm{Hl}, \mathrm{Od}$, and Ph. Cynoscion similis was differentiated from the closely related $C$. jamaicensis based on the analysis of the factor loadings for the first and second principal components. Meristic ranges are presented for Cynoscion similis and $C$. jamaicensis. In the text, measurements are given as percentages of the standard length (SL), unless otherwise stated. The diagnosis of the western Atlantic species was based on direct examination of specimens. The diagnostic characters of the western Atlantic species in relation to species from the eastern Pacific was based on the literature (Chao 1978, 1995, 2003; Castro-Aguirre 1999; McEachran and Fechhelm 2005) and the Guide to the shore fishes of the Caribbean and adjacent areas of the Smithsonian Tropical Research Institute (www. stri.org/ sfgc and www.stri.org/sftep).

\section{Molecular data}

Partial COI sequences (652 bp) were obtained from five specimens of Cynoscion similis. Additionally, sequences were generated for two specimens of Cynoscion jamaicensis, and 73 sequences for 11 Cynoscion species were obtained from GenBank [C. acoupa (4), C. arenarius (6), C. guatuculpa (20), C. leiarchus (4), C. nebulosus (3), C. microlepidotus (4), C. nothus (2), C. jamaicensis (9), C. regalis (4), C. similis (1) and C. virescevs (16)] (Table 1). Vouchers and tissues of the specimens sequenced here were deposited in the fish collection of the Laboratory for Fish Biology and Genetics (LBP) of the Department of Morphology of Universidade Estadual Paulista (UNESP) in Botucatu, São Paulo, Brazil and Museu Paraense Emílio Goeldi (MPEG) in Belém, Pará, Brazil.
Table 1. Genbank number of sequences used and added (*) in the present study. Discrepancies of species identification with GenBank are indicated in bold.

\begin{tabular}{|c|c|c|c|}
\hline \multicolumn{2}{|c|}{ Species identification } & \multirow{2}{*}{ Locality } & \multirow{2}{*}{$\begin{array}{c}\text { GenBank } \\
\text { Accession } \\
\text { Number }\end{array}$} \\
\hline Present study & GenBank & & \\
\hline \multirow[t]{4}{*}{$\begin{array}{l}\text { Cynoscion } \\
\text { acoupa }\end{array}$} & Cynoscion acoupa & São Paulo, Brazil & JQ365312 \\
\hline & & Buenos Aires, Argentina & KP722713 \\
\hline & & Pará, Brazil & KP331710 \\
\hline & $\begin{array}{l}\text { Cynoscion } \\
\text { nebulosus }\end{array}$ & Florida, United States & JN021296 \\
\hline \multirow[t]{6}{*}{$\begin{array}{l}\text { Cynoscion } \\
\text { arenarius }\end{array}$} & $\begin{array}{l}\text { Cynoscion } \\
\text { arenarius }\end{array}$ & Florida, United States & JQ841862 \\
\hline & & Alabama, United States & KF461162 \\
\hline & & Alabama, United States & KF461163 \\
\hline & & Alabama, United States & KF461164 \\
\hline & & Alabama, United States & KF461165 \\
\hline & Cynoscion & Florida, United States & JQ841861 \\
\hline \multirow[t]{20}{*}{$\begin{array}{l}\text { Cynoscion } \\
\text { guatucupa }\end{array}$} & $\begin{array}{l}\text { Cynoscion } \\
\text { guatucupa }\end{array}$ & São Paulo, Brazil & GU702425 \\
\hline & & São Paulo, Brazil & GU702426 \\
\hline & & São Paulo, Brazil & GU702427 \\
\hline & & São Paulo, Brazil & GU702428 \\
\hline & & São Paulo, Brazil & GU702485 \\
\hline & & São Paulo, Brazil & GU702486 \\
\hline & & São Paulo, Brazil & GU702487 \\
\hline & & São Paulo, Brazil & GU702488 \\
\hline & & São Paulo, Brazil & GU702490 \\
\hline & & São Paulo, Brazil & JQ365313 \\
\hline & & São Paulo, Brazil & JQ365314 \\
\hline & & São Paulo, Brazil & JQ365315 \\
\hline & & São Paulo, Brazil & JQ365316 \\
\hline & & Buenos Aires, Argentina & KP722714 \\
\hline & & Buenos Aires, Argentina & EU074395 \\
\hline & & Buenos Aires, Argentina & EU074396 \\
\hline & & Buenos Aires, Argentina & EU074397 \\
\hline & & Buenos Aires, Argentina & EU074398 \\
\hline & & Buenos Aires, Argentina & EU074399 \\
\hline & $\begin{array}{c}\text { Cynoscion } \\
\text { steindachneri }\end{array}$ & not described & KP331709 \\
\hline \multirow[t]{11}{*}{$\begin{array}{l}\text { Cynoscion } \\
\text { jamaicensis }\end{array}$} & $\begin{array}{l}\text { Cynoscion } \\
\text { jamaicensis }\end{array}$ & Rio de Janeiro, Brazil & $J \times 124767$ \\
\hline & & Rio de Janeiro, Brazil & $J \times 124768$ \\
\hline & & São Paulo, Brazil & JQ365317 \\
\hline & & São Paulo, Brazil & JQ365318 \\
\hline & & Pará, Brazil & MH575181* \\
\hline & & Pará, Brazil & MH575182* \\
\hline & & Pará, Brazil & KP331711 \\
\hline & & Alagoas, Brazil & KY402377 1 \\
\hline & & Alagoas, Brazil & KY402378 1 \\
\hline & & Alagoas, Brazil & KY402379 \\
\hline & & Alagoas, Brazil & KY402380 \\
\hline
\end{tabular}


Table 1. Continued.

\begin{tabular}{|c|c|c|c|}
\hline \multicolumn{2}{|c|}{ Species identification } & \multirow{2}{*}{ Locality } & \multirow{2}{*}{$\begin{array}{c}\text { GenBank } \\
\text { Accession } \\
\text { Number }\end{array}$} \\
\hline Present study & GenBank & & \\
\hline \multirow[t]{4}{*}{$\begin{array}{l}\text { Cynoscion } \\
\text { leiarchus }\end{array}$} & $\begin{array}{l}\text { Cynoscion } \\
\text { leiarchus }\end{array}$ & Bahia, Brazil & BAHIA440 15 \\
\hline & & Bahia, Brazil & BAHIA441 15 \\
\hline & & São Paulo, Brazil & JQ365319 \\
\hline & & Belize, Guatemala & KF929804 \\
\hline \multirow[t]{4}{*}{$\begin{array}{c}\text { Cynoscion } \\
\text { microlepidotus }\end{array}$} & $\begin{array}{l}\text { Cynoscion } \\
\text { microlepidotus }\end{array}$ & Pará, Brazil & KP331702 \\
\hline & & Pará, Brazil & KP331713 \\
\hline & & São Paulo, Brazil & JQ365320 \\
\hline & Cynoscion sp. & Brazil & HQ689365 \\
\hline \multirow[t]{3}{*}{$\begin{array}{l}\text { Cynoscion } \\
\text { nebulosus }\end{array}$} & $\begin{array}{l}\text { Cynoscion } \\
\text { nebulosus }\end{array}$ & Alabama, United States & KF461166 \\
\hline & & Alabama, United States & KF461167 \\
\hline & & Florida, United States & JN021296 \\
\hline \multirow[t]{2}{*}{$\begin{array}{l}\text { Cynoscion } \\
\text { nothus }\end{array}$} & Cynoscion nothus & Florida, United States & JQ841863 \\
\hline & & Florida, United States & JQ841864 \\
\hline \multirow[t]{4}{*}{$\begin{array}{c}\text { Cynoscion } \\
\text { regalis }\end{array}$} & Cynoscion regalis & Virginia, United States & KT075323 \\
\hline & & Florida, United States & KP722717 \\
\hline & & United States & KT075305 \\
\hline & & United States & KF929807 \\
\hline \multirow[t]{6}{*}{$\begin{array}{c}\text { Cynoscion } \\
\text { similis }\end{array}$} & Cynoscion similis & Pará, Brazil & MH575183* \\
\hline & & Pará, Brazil & MH575184* \\
\hline & & Pará, Brazil & MH575185* \\
\hline & & Pará, Brazil & MH575186* \\
\hline & & Pará, Brazil & MH575187* \\
\hline & $\begin{array}{c}\text { Cynoscion } \\
\text { jamaicensis }\end{array}$ & Pará, Brazil & KP331706 \\
\hline \multirow[t]{16}{*}{$\begin{array}{l}\text { Cynoscion } \\
\text { virescens }\end{array}$} & $\begin{array}{l}\text { Cynoscion } \\
\text { virescens }\end{array}$ & Pará, Brazil & KP331712 1 \\
\hline & & São Paulo, Brazil & JQ365321 \\
\hline & & São Paulo, Brazil & JQ36532 \\
\hline & & São Paulo, Brazil & HM424137 \\
\hline & & Alagoas, Brazil & KY402371 \\
\hline & & Alagoas, Brazil & KY402372 \\
\hline & & Alagoas, Brazil & KY402373 \\
\hline & & Alagoas, Brazil & KY402374 \\
\hline & & Brazil & HQ689366 \\
\hline & & Brazil & HQ689367 \\
\hline & & Brazil & HQ689368 \\
\hline & & Brazil & HQ689369 \\
\hline & & Brazil & HQ689370 \\
\hline & & Brazil & HQ689371 \\
\hline & & Brazil & HQ689372 \\
\hline & & Brazil & HQ689373 \\
\hline
\end{tabular}

\section{DNA extraction and sequencing}

Genomic DNA was isolated from fins or muscle tissues of each specimen with a DNeasy Blood and Tissue Kit (Qiagen, cat. 69506), according to the manufacturer instructions. Amplifications were performed in a total volume of 12.5 $\mu \mathrm{l}$ with $1.25 \mu \mathrm{l}$ of $10 \mathrm{X}$ buffer $(10 \mathrm{mM}$ Tris $-\mathrm{HCl}+15 \mathrm{mM}$ $\mathrm{MgCl} 2), 0.5 \mu \mathrm{dNTPs}(200 \mathrm{nM}$ of each), $0.5 \mu \mathrm{l}$ each $5 \mathrm{mM}$ primer (FishF2, FishR2 described in Ward et al. 2005), 0.05 $\mu$ Platinum ${ }^{\circ}$ Taq Polymerase (Invitrogen -5 units $-\mu \mathrm{l}$ ), 1 $\mu l$ template DNA (12 ng), and $8.7 \mu \mathrm{ddH} 2 \mathrm{O}$. The PCR reactions consisted of $30-40$ cycles, $30 \mathrm{~s}$ at $95^{\circ} \mathrm{C}, 15-30 \mathrm{~s}$ at $48-54^{\circ} \mathrm{C}$ (according to each species), and $45 \mathrm{~s}$ at $72{ }^{\circ} \mathrm{C}$. All PCR products were first visually identified on a $1 \%$ agarose gel and then purified using ExoSap - IT ${ }^{\oplus}$ (USB Corporation) following manufacturer instructions. The purified PCR products were sequenced using the "Big DyeTM Terminator v 3.1 Cycle Sequencing Ready Reaction Kit" (Applied Biosystems), purified again by ethanol precipitation and loaded on an automatic sequencer 3130 - Genetic Analyzer (Applied Biosystems).

\section{Sequencing analysis}

Consensus sequences from forward and reverse strands were obtained using Geneious Pro 8.1.8 (Kearse et al. 2012). Alignments were generated using MUSCLE algorithm (Edgar 2004) under default parameters. After alignment, the matrix was checked by eye for any obvious misalignments, and to detect potential cases of sequencing errors. A quality control step was included in our workflow to detect contamination, paralogous copies or pseudogenes. After that, the presence of stop codons was checked using Geneious. Nucleotide variation, substitution patterns and genetic distances were examined using Mega 6.06 (Tamura et al. 2013).

Maximum likelihood (ML) analyses were generated in a partitioned RAxML analysis using the CIPRES web server (Miller et al. 2010). Random starting trees were used for ML tree search and all other parameters were set to default values. All ML analyses were performed under $G T R+G$ since RAxML only applies to this model (Stamatakis et al. 2008). The robustness of the topology was investigated using 1000 bootstrap pseudoreplicates (Felsenstein 1985).

\section{RESULTS}

Cynoscion similis Randall \& Cervigón, 1968

Figures 1 and 2, Tables 2 and 3

Cynoscion simlis - Randall and Cervigón 1968: 179 (original description; type locality: Isla de Margarita, Venezuela) - Lasso et al. 1999: 11 (type catalog: collection of Fundación La Salle; listed) - Chao 1978: 36 (basis for classifying western Atlantic sciaenids; listed. short description) - Uyeno et al. 1983: 371 (fishes trawled off Suriname and French Guiana; listed; photograph and short description) - Cervigón 1992: 401 
(FAO sheets of marine fishes in septentrional South America; listed) - Cervigón 1993: 261 (marine fishes of Venezuela; listed and description) - Aguillera 1998: 50 (marine fishes of Venezuela; listed) - Chao 2002: 10 (Cynoscion; taxonomy; list and identification key) - Chao 2003: 1614 (living marine resources of the western Central Atlantic; list; short description; illustration) - Casatti and Menezes 2003: 86 (catalog of marine fishes from Brazil; listed) - Chao and Aguillera 2015 (IUCN Redlist; biology and distribution).

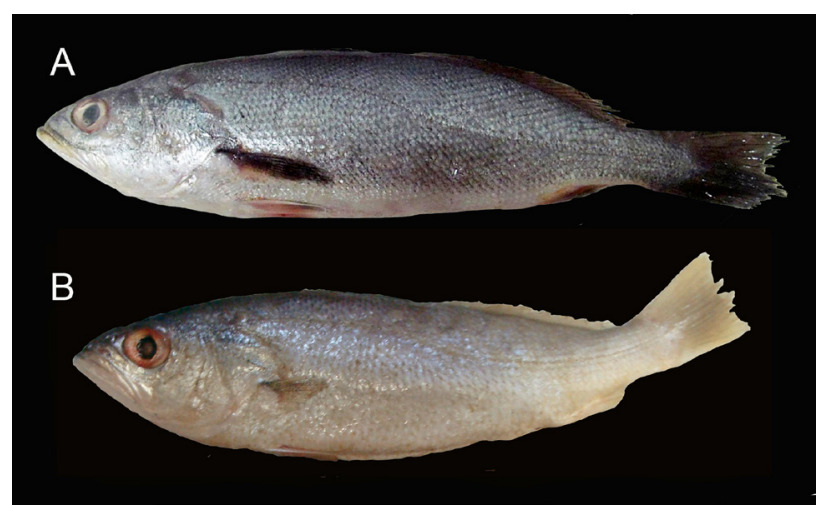

Figure 1. Body in lateral view. A. Cynoscion similis (MPEG 35679, 304 mm SL). B. Cynoscion jamaicensis (MPEG 35508, $184 \mathrm{~mm} \mathrm{SL).} \mathrm{This} \mathrm{figure} \mathrm{is} \mathrm{in} \mathrm{color} \mathrm{in} \mathrm{the}$ electronic version.

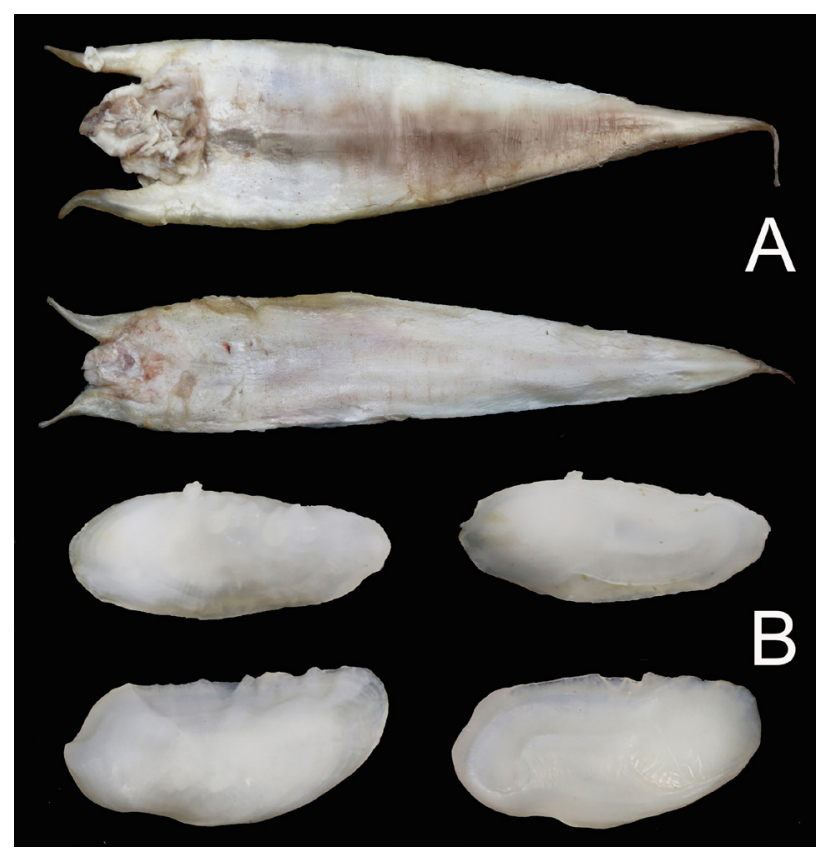

Figure 2. Cynoscion similis (above, AZUSC 5709, $388 \mathrm{~mm} \mathrm{SL}$ ) and C. jamaicensis (below, AZUSC 2937, 326 mm SL). (A) Swimbladder and (B) Sagitta otoliths (left and right side). This figure is in color in the electronic version.
Table 2. Selected meristic characters of Cynoscion similis and C. jamaicensis, Underlined values refer to type specimens. Lateral line scales $(\mathbf{A})$; dorsal-fin rays $(\mathbf{B})$; pectoral-fin rays $(\mathbf{C})$; anal-fin rays $(\mathbf{D})$; gill rakers on the first gill arch $(\mathbf{E})$; gill rakers on the second gill arch $(\mathbf{F})$.

\begin{tabular}{lccccccc}
\hline A & $\mathbf{5 1}$ & $\mathbf{5 2}$ & $\mathbf{5 3}$ & $\mathbf{5 4}$ & $\mathbf{5 5}$ & $\mathbf{5 6}$ & $\mathbf{5 7}$ \\
C. similis & & 1 & $\underline{5}$ & $\underline{7}$ & $\underline{7}$ & $\underline{4}$ & $\underline{2}$ \\
C. jamaicensis & 1 & 5 & 5 & 19 & 9 & & \\
\hline B & $\mathbf{2 2}$ & $\mathbf{2 3}$ & $\mathbf{2 4}$ & $\mathbf{2 5}$ & $\mathbf{2 6}$ & $\mathbf{2 7}$ & $\mathbf{2 8}$ \\
C. similis & & & 1 & $\underline{7}$ & $\underline{21}$ & $\underline{7}$ & 1 \\
C. jamaicensis & 1 & 13 & 22 & 15 & 5 & & \\
\hline C & $\mathbf{1 5}$ & $\mathbf{1 6}$ & $\mathbf{1 7}$ & $\mathbf{1 8}$ & $\mathbf{1 9}$ & & \\
C. similis & & & $\underline{4}$ & $\underline{23}$ & 5 & & \\
C. jamaicensis & 2 & 6 & 41 & 13 & & & \\
\hline D & $\mathbf{8}$ & $\mathbf{9}$ & $\mathbf{1 0}$ & & & & \\
C. similis & 2 & $\underline{25}$ & 6 & & & & \\
C. jamaicensis & 1 & 23 & 5 & & & & \\
\hline E & $\mathbf{1 1}$ & $\mathbf{1 2}$ & $\mathbf{1 3}$ & $\mathbf{1 4}$ & $\mathbf{1 5}$ & $\mathbf{1 6}$ & $\mathbf{1 7}$ \\
C. similis & 4 & 6 & 3 & 8 & & & \\
C. jamaicensis & & & 4 & 5 & 13 & 6 & 1 \\
\hline F & $\mathbf{7}$ & $\mathbf{8}$ & $\mathbf{9}$ & $\mathbf{1 0}$ & $\mathbf{1 1}$ & $\mathbf{1 2}$ & $\mathbf{1 3}$ \\
C. similis & & & 3 & 5 & 4 & 1 & 3 \\
C. jamaicensis & 4 & 5 & 5 & 5 & 6 & 3 & 1 \\
\hline & & & & & & &
\end{tabular}

Table 3. Measurements of Cynoscion similis (A. holotype, B. paratypes) and Cynoscion jamaicensis expressed as percentages of standard length. Morphometric characters suitable to discriminate the two species are indicated in bold. All measures in $\mathrm{mm}$.

\begin{tabular}{|c|c|c|c|c|c|c|c|c|}
\hline \multirow{2}{*}{$\begin{array}{l}\text { Morphometric } \\
\text { character }\end{array}$} & \multicolumn{5}{|c|}{ Cynoscion similis } & \multicolumn{3}{|c|}{ Cynoscion jamaicensis } \\
\hline & $A$ & $B$ & $\mathrm{~N}$ & Mean & Range & $\mathrm{N}$ & Mean & Range \\
\hline $\begin{array}{l}\text { Standard } \\
\text { length }\end{array}$ & 284 & $346-482$ & 22 & & $213-309$ & 29 & & $142-285$ \\
\hline Head length & 32.4 & $29.5-32.6$ & 21 & 31.9 & $30.1-34.0$ & 29 & 31.7 & $27.2-35.5$ \\
\hline Head depth & & & 22 & 24.7 & $23.0-26.2$ & 29 & 24.4 & $22.9-27.6$ \\
\hline $\begin{array}{l}\text { Pos-orbital } \\
\text { length }\end{array}$ & 19.0 & $18.2-19.8$ & 22 & 18.7 & $17.8-19.7$ & 29 & 16.9 & $15.5-18.9$ \\
\hline $\begin{array}{l}\text { Orbital } \\
\text { diameter }\end{array}$ & & & 22 & 7.0 & $6.4-7.5$ & 29 & 8.6 & $7.7-9.3$ \\
\hline $\begin{array}{l}\text { Interorbital } \\
\text { distance }\end{array}$ & 6.4 & $5.8-6.3$ & 22 & 6.5 & $5.6-7.5$ & 29 & 7.2 & $6.6-8.2$ \\
\hline Snout length & 8.3 & $7.5-8.4$ & 21 & 8.1 & $7.6-8.5$ & 29 & 8.6 & $7.8-9.1$ \\
\hline Mouth width & & & 22 & 10.7 & $9.2-12.3$ & 28 & 8.3 & $7.0-9.2$ \\
\hline $\begin{array}{l}\text { Maxillary } \\
\text { length }\end{array}$ & 6.7 & $4.2-4.9$ & 21 & 4.5 & $3.7-5.0$ & 29 & 4.8 & $4.2-5.5$ \\
\hline $\begin{array}{l}\text { Maxillary } \\
\text { height }\end{array}$ & 14.4 & $13.0-13.8$ & 22 & 14.3 & $13.6-14.7$ & 28 & 14.6 & $14.0-15.5$ \\
\hline Body depth & 24.1 & $23.4-26.7$ & 22 & 26.3 & $24.9-27.5$ & 28 & 24.3 & $22.9-26.0$ \\
\hline Body width & & & 22 & 16.0 & $13.6-17.5$ & 29 & 14.9 & $12.0-17.4$ \\
\hline $\begin{array}{l}\text { Distance } \\
\text { from snout to } \\
\text { dorsal fin }\end{array}$ & 34.8 & $32.9-35.1$ & 22 & 34.6 & $33.9-35.9$ & 27 & 36.1 & $34.8-37.8$ \\
\hline $\begin{array}{l}\text { Dorsal-fin } \\
\text { length }\end{array}$ & 15.5 & $13.0-14.9$ & 22 & 13.3 & $12.2-15.0$ & 29 & 14.8 & $11.8-17.5$ \\
\hline $\begin{array}{l}\text { Dorsal-fin } \\
\text { base length }\end{array}$ & 53.7 & $51.2-55.3$ & 22 & 54.0 & $51.6-57.1$ & 27 & 52.1 & $48.9-54.3$ \\
\hline $\begin{array}{l}\text { Distance } \\
\text { from snout to } \\
\text { pectoral fin }\end{array}$ & & & 21 & 29.9 & $29.1-31.2$ & 29 & 30.8 & $25.9-31.7$ \\
\hline $\begin{array}{l}\text { Pectoral fin } \\
\text { length }\end{array}$ & 20.1 & $18.8-20.8$ & 22 & 17.9 & $15.8-19.6$ & 17 & 16.5 & $11.4-20.6$ \\
\hline
\end{tabular}


Table 3. Continued.

\begin{tabular}{|c|c|c|c|c|c|c|c|c|}
\hline \multirow{2}{*}{$\begin{array}{l}\text { Morphometric } \\
\text { character }\end{array}$} & \multicolumn{5}{|c|}{ Cynoscion similis } & \multicolumn{3}{|c|}{ Cynoscion jamaicensis } \\
\hline & A & B & $\mathrm{N}$ & Mean & Range & $\mathrm{N}$ & Mean & Range \\
\hline Distance from & & & & & & & & \\
\hline $\begin{array}{l}\text { snout to pelvic } \\
\text { fin }\end{array}$ & 33.3 & $32.0-34.5$ & 22 & 30.7 & $29.2-32.2$ & 29 & 32.1 & $30.0-34.3$ \\
\hline $\begin{array}{l}\text { Pelvic fin } \\
\text { length }\end{array}$ & 16.5 & $15.3-17.6$ & 22 & 14.4 & $13.0-16.2$ & 29 & 15.2 & $11.0-19.4$ \\
\hline Distance from & & & & & & & & \\
\hline $\begin{array}{l}\text { snout to anal } \\
\text { fin }\end{array}$ & 75.8 & $71.3-76.7$ & 16 & 74.2 & $71.6-76.7$ & 26 & 76.4 & $73.7-79.6$ \\
\hline $\begin{array}{l}\text { Anal-fin } \\
\text { length }\end{array}$ & 12.5 & $11.2-12.0$ & 21 & 11.0 & $9.5-12.8$ & 28 & 11.2 & $8.9-13.8$ \\
\hline $\begin{array}{l}\text { Anal-fin base } \\
\text { length }\end{array}$ & 8.9 & $7.9-10.2$ & 22 & 8.8 & $7.7-10.2$ & 29 & 9.0 & $8.1-11.4$ \\
\hline $\begin{array}{l}\text { Caudal- } \\
\text { peduncle } \\
\text { height }\end{array}$ & 8.8 & $7.4-8.1$ & 22 & 8.4 & $7.8-8.9$ & 28 & 9.8 & $8.9-10.5$ \\
\hline $\begin{array}{l}\text { Caudal- } \\
\text { peduncle } \\
\text { length }\end{array}$ & 18.0 & $17.6-19.5$ & 22 & 18.1 & $16.5-20.5$ & 29 & 16.6 & $14.2-19.5$ \\
\hline
\end{tabular}

\section{Morphological diagnosis}

Cynoscion similis is distinguished from its western Atlantic congeners as follows: from C. acoupa, by having $24-29 \mathrm{soft}$ dorsal rays (vs. $17-23)$, pelvic fin much shorter than pectoral fin (vs. pelvic fin nearly as long as pectorals), rear tail margin straight to slightly concave (vs. rhomboidal), pectoral, anal, and caudal fins black ( $v$ s. yellowish orange), and dense black coloration of inner margin of operculum (vs. dusky); from $C$. arenarius, by having anal and caudal fins black (vs. yellowish), and interior of operculum black (vs. dusky), anal soft rays 8 10 (vs. 10 - 12, usually 11); from C. guatucupa, by having 24 - 29 soft dorsal rays (vs. 18 -21), and absence of marked dark stripes or spots on flanks (vs. flanks with marked dark stripes or spots); gill rakers 11 - 14 (vs. 21 - 16); from C. leiarchus, by having 24 - 29 soft dorsal rays (vs. 20 - 24), less than 100 transversal rows of scales on trunk ( $v s$. more than 100 rows), presence of ctenoid scales on trunk (vs. cycloid scales), pelvic fin much shorter than pectoral fin (vs. pelvic fin larger than pectoral fin), anal fin black ( $v s$. yellowish), and dense black coloration in inner operculum margin (vs. dusky); from $C$. microlepidotus, by having less than 100 transversal rows of scales on trunk (vs. more than 100 rows), presence of ctenoid scales on trunk (vs. cycloid scales), pelvic fin much shorter than pectoral fin ( $v s$. pelvic fin larger than pectoral fin), rear tail margin truncated ( $v s$. rhomboidal), and pectoral, anal and caudal fins black (vs. yellowish), scales on lateral line $52-57$ (vs. 65 - 72); from C. nebulosus, by pelvic fin much shorter than pectoral fin (vs. pelvic fin longer than pectoral fin), and body with no marked round black spots near dorsal fin margin (vs. numerous round black spots scattered irregularly on upper half of body); from C. nothus, by having pectoral, anal and caudal fins black (vs. yellowish); from $C$. regalis, by back with oblique dotted lines along scale rows ( $v$ s. back with small dark spots forming undulating oblique dotted or reticulated lines), and anal fin black (vs. yellowish or pale), gill rakers $11-14$ (vs. 14 - 17), anal soft rays 8 - 10 (vs. 10 - 13, usually 12); from $C$. virescens, by presence of ctenoid scales on trunk (vs. cycloid scales), less than 100 transversal rows of scales on trunk (vs. more than 100 rows), pectoral and anal fins black (vs. yellowish to orangish), and rear margin of tail straight to slightly concave ( $v s$. pointed in juvenile, with asymmetric blunt point in adult), dorsal soft rays $24-29$ (vs. 27 - 31); C. steindachneri, by truncated caudal fin (vs. rhomboidal caudal fin), dorsal soft rays $24-29$ (vs. $21-24)$, anal soft rays 8 - 10 (vs. 10 - 12).

Cynoscion similis is most similar to C. jamaicensis, but both species were clearly distinguished in the Principal Component Analysis (PCA) in relation to the most prominent character traits (Figure 3). The two principal axes of the PCA together explained $85.6 \%$ of the variation in the morphometric characters. The first axis was related positively to Od, SDd, $\mathrm{CPh}, \mathrm{IOd}, \mathrm{SPd}, \mathrm{SL}, \mathrm{Mh}$, and negatively to $\mathrm{Mw}$ and $\mathrm{POl}$ (Figure 3). The second axis was related positively to SPd, Pol, $\mathrm{Mh}, \mathrm{IOd}, \mathrm{Sl}$ and negatively to $\mathrm{Mw}, \mathrm{CPh}, \mathrm{SDh}$, Od (Figure 3). Otherwise Cynoscion similis can be differentiated by having black pectoral and anal fins (vs. yellowish); soft dorsal fin barely covered with 1 - 2 series of scales (vs. dorsal fin covered with scales along more than half its length); pectoral fin usually with 18 rays (vs. usually $16-17$ rays in pectoral fin); rear maxillary border slender, rounded, reaching vertical that pass over posterior eye border ( $v s$. truncate, not reaching vertical through rear eye border); mouth width $9.2-12.3 \%$ of SL ( $v$. $7.0-9.2 \%$ of SL); and caudal peduncle depth $7.8-8.9 \%$ of SL (vs. $8.9-10.5 \%$ of SL).

\section{Molecular diagnosis}

The DNA barcoding showed that Cynoscion similis formed a distinct cluster (Figure 4) with genetic distances (K2P) from the remaining western Atlantic species, ranginng from $8.1 \%$ (C. jamaicensis) to $15.6 \%$ (C. guatucupa) (Table 4). Cynoscion similis haplotypes differed from those of its congeners by 49 (C. jamaicensis) to 102 bases (C. guatucupa) (Supplementary Material, Appendix S1).

\section{Description}

Dorsal fin rays $\mathrm{X}+\mathrm{I} .24-28$; anal fin rays II.8-10; pectoral fin rays 17-19; lateral line scales 52-57; scales above lateral line 7-8, below lateral line 11-13; transverse rows of scales from base of pectoral fin to caudal peduncle 68-79; developed gill rakers $2-3+8-11,11-14$ in 1 st arch, 9-13 in 2 nd arch.

Snout pointed, nearly equal to eye length; dorsal profile convex from upper jaw to dorsal fin origin; slightly concave on dorsal margin, immediately anterior to eye. A pair of small, slender pores on the lower jaw separated by symphysis, pores otherwise not visible even under stereomicroscope. Mouth large, terminal, cleft angle when closed $45^{\circ}$; lower jaw prognathous; maxillary reaching vertical at posterior margin of eye. Lower jaw heavy; mental process prominent. Lips slender. 

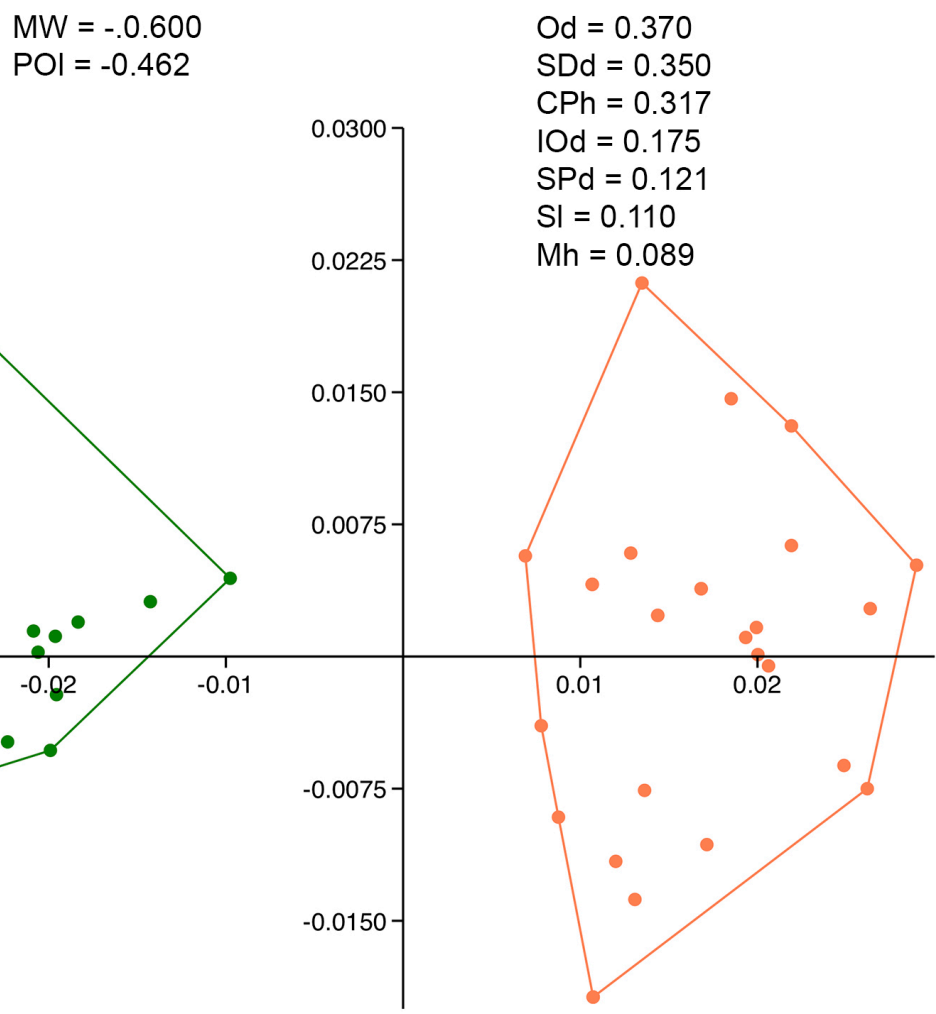

$\mathrm{Mw}=-0.393$

$\mathrm{CPh}=-0.195$

$\mathrm{SDh}=-0.181$

Od $=-0.142$

Component 1

Figure 3. Principal components analysis loadings (PC1 and PC2) for nine morphometric variables of Cynoscion similis (green) and Cynoscion jamaicensis (orange), with factor loadings for the first and second principal components. Abbreviations: $\mathrm{Kph}=$ Caudal - peduncle height; IOd = Interorbital distance; $\mathrm{Mh}=\mathrm{Maxillary}$ height; $\mathrm{Mw}$ $=$ Mouth width; $\mathrm{Od}=$ Orbital diameter; POI = Post - orbital length; SDd = Distance from snout to dorsal fin; SPd = Distance from the tip of the snout to the pectoral fin; $\mathrm{SI}=$ Snout length. This figure is in color in the electronic version.

Table 4. K2P genetic pairwise distance (mean \pm standard error) between Cynoscion species. Values in bold on the diagonal are the intraspecific distances.

\begin{tabular}{|c|c|c|c|c|c|c|c|}
\hline & & 1 & 2 & 3 & 4 & 5 & 6 \\
\hline 1 & C. acoupa & $0.003 \pm 0.002$ & & & & & \\
\hline 2 & C. arenarius & $0.087 \pm 0.011$ & $0.002 \pm 0.001$ & & & & \\
\hline 3 & C. guatucupa & $0.147 \pm 0.015$ & $0.147 \pm 0.013$ & $0.008 \pm 0.002$ & & & \\
\hline 4 & C. jamaicensis & $0.091 \pm 0.012$ & $0.095 \pm 0.012$ & $0.155 \pm 0.015$ & $0.001 \pm 0.000$ & & \\
\hline 5 & C. leiarchus & $0.147 \pm 0.014$ & $0.159 \pm 0.015$ & $0.158 \pm 0.015$ & $0.128 \pm 0.014$ & $0.003 \pm 0.001$ & \\
\hline 6 & C. microlepidotus & $0.157 \pm 0.015$ & $0.163 \pm 0.016$ & $0.169 \pm 0.016$ & $0.126 \pm 0.014$ & $0.145 \pm 0.015$ & $0.000 \pm 0.000$ \\
\hline 7 & C. nebulosus & $0.109 \pm 0.013$ & $0.103 \pm 0.013$ & $0.150 \pm 0.015$ & $0.105 \pm 0.013$ & $0.147 \pm 0.015$ & $0.160 \pm 0.017$ \\
\hline 8 & C. nothus & $0.092 \pm 0.012$ & $0.091 \pm 0.011$ & $0.155 \pm 0.014$ & $0.094 \pm 0.012$ & $0.139 \pm 0.014$ & $0.152 \pm 0.016$ \\
\hline 9 & C. regalis & $0.098 \pm 0.012$ & $0.060 \pm 0.010$ & $0.149 \pm 0.014$ & $0.107 \pm 0.013$ & $0.154 \pm 0.015$ & $0.170 \pm 0.015$ \\
\hline 10 & C. similis & $0.104 \pm 0.012$ & $0.104 \pm 0.012$ & $0.156 \pm 0.015$ & $0.081 \pm 0.011$ & $0.154 \pm 0.015$ & $0.147 \pm 0.015$ \\
\hline \multirow[t]{2}{*}{11} & C. virescens & $0.133 \pm 0.016$ & $0.139 \pm 0.016$ & $0.157 \pm 0.017$ & $0.120 \pm 0.015$ & $0.146 \pm 0.016$ & $0.160 \pm 0.018$ \\
\hline & & 7 & 8 & & & 10 & 11 \\
\hline 1 & C. acoupa & & & & & & \\
\hline 2 & C. arenarius & & & & & & \\
\hline 3 & C. guatucupa & & & & & & \\
\hline 4 & C. jamaicensis & & & & & & \\
\hline 5 & C. leiarchus & & & & & & \\
\hline 6 & C. microlepidotus & & & & & & \\
\hline 7 & C. nebulosus & $0.003 \pm 0.002$ & & & & & \\
\hline 8 & C. nothus & $0.097 \pm 0.012$ & $0.003 \pm 0.002$ & & & & \\
\hline 9 & C. regalis & $0.108 \pm 0.013$ & $0.096 \pm 0.011$ & & .000 & & \\
\hline 10 & C. similis & $0.112 \pm 0.013$ & $0.094 \pm 0.012$ & & .012 & $1 \pm 0.001$ & \\
\hline 11 & C. virescens & $0.148 \pm 0.017$ & $0.138 \pm 0.016$ & & .016 & $1 \pm 0.015$ & $0.005 \pm 0.002$ \\
\hline
\end{tabular}




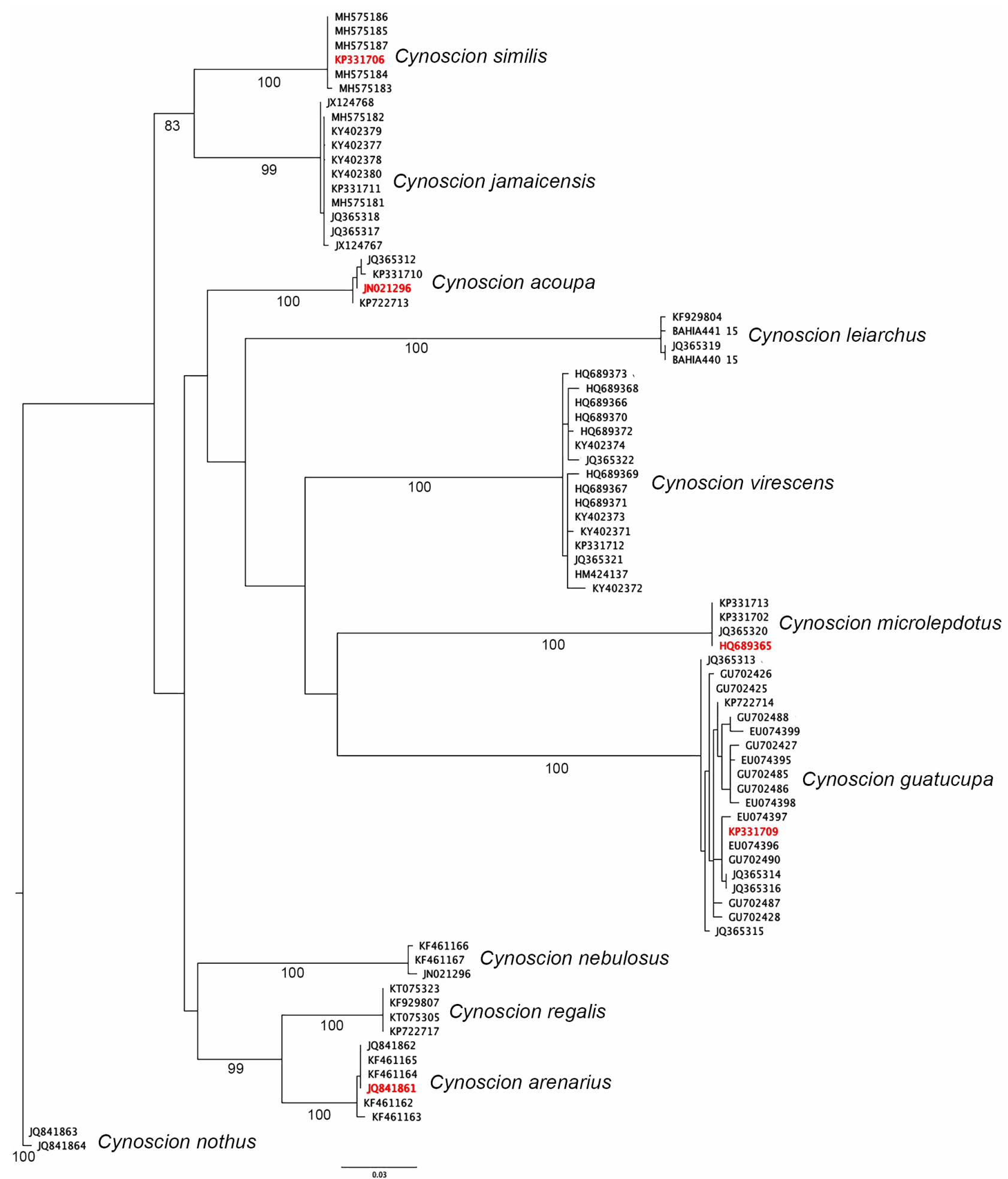

Figure 4. Maximum likelihood tree of the species of Cynoscion based on partial sequences of the cytochrome oxidase c subunit I. The numbers at the nodes correspond to the results of the bootstrap analysis. Discrepancies of species identification in GenBank are indicated in red (see Table 1). 
Upper jaw with two series of caniniform teeth (on inner row largest); 2 stout fangs nearby tip of upper jaw about half size of pupil; lower jaw with two rows of caniniform teeth, one series posteriorly with 5-8 larger teeth about size of posterior nostril. Eye large, diameter slightly greater than interorbital width. Anterior and posterior nostrils separated, anterior one small, oval, second about 2 times larger, elongate or teardrop-shaped, almost half size of pupil. Distance between nares slightly smaller than first nostril. Gill rakers stout, without spines, longest one about half eye size. Scales on head and body. Head with cycloid scales on interorbital region (posterior to that in smaller specimens to $173 \mathrm{~mm} \mathrm{SL}$ ), nape, chin (about 10 vertical rows), opercle (about 15 vertical rows), posterior part of infraorbital region (naked in smaller specimens), ctenoid on belly, abdomen and flanks. Scales absent on dorsal and anal fins except for basal 2 or 3 rows. Pectoral fins much longer than pelvic fins, reaching pelvic fin tips, but not vent. First anal fin spine, second one shorter than longer anal fin rays. Caudal fin tip concave.

Gas bladder long, carrot-shaped, similar to that of $C$. jamaicensis (Figure 2a), but less slender, with a pair of lateral hornlike stout appendages on the anterior border of bladder. Sagitta more slender than in C. jamaicensis (Figure 2b), profile of the upper margin slightly convex, with a small fingerlike projection; lower margin convex with a tiny medial bump, anterior border convex, blunt, or with evident anterodorsal concavity.

\section{Color in life}

Fresh specimens are silvery with slender dark brown lines along scale rows on the flanks and upper half (Figure 1a). Snout and lower jaw tip dark, mouth aperture pale with dusky margin near lower jaw. Upper half of pectoral fin with dense black, black spot over pectoral fin, caudal fin rays black, fins otherwise dusky, more so in the first spine of the pelvic fin. Inner operculum margin black, with pale rear border.

\section{Distribution}

Cynoscion similis is endemic from the Orinoco-Amazon Plume, with records from Trinidad and Tobago (ROM 61788, CAS 56814), Venezuela (USNM 201382, USNM 233071), French Guiana (UF 204299, USNM 325179), Suriname (UF 212502, USNM 184911), and the Brazilian north coast in the states of Amapá (CAS 48885, USNM 307229, USNM 325182, USNM 325184, and material examined in the present study) and Pará (material examined in the present study) (Figure 5).

\section{Biology}

There is a wealth of studies on the biology of the other Cynoscion species of the Atlantic Ocean (Santos and Vianna 2017), but no information is available specifically for $C$. similis.

\section{Habits}

Randall and Cervigón (1968) described C. similis based on specimens collected at depths of 10 to 30 fathoms (18-54 $\mathrm{m})$, but the specimens we examined were collected in deeper waters $(40-70 \mathrm{~m})$, over sandy or muddy bottoms.

\section{Remarks}

Randall and Cervigón (1968) described 2-3+7-8 rakers (9-11) on the first gill arch of Cynoscion similis. Our specimens had a larger number of gill rakers (11-14), but there is compelling

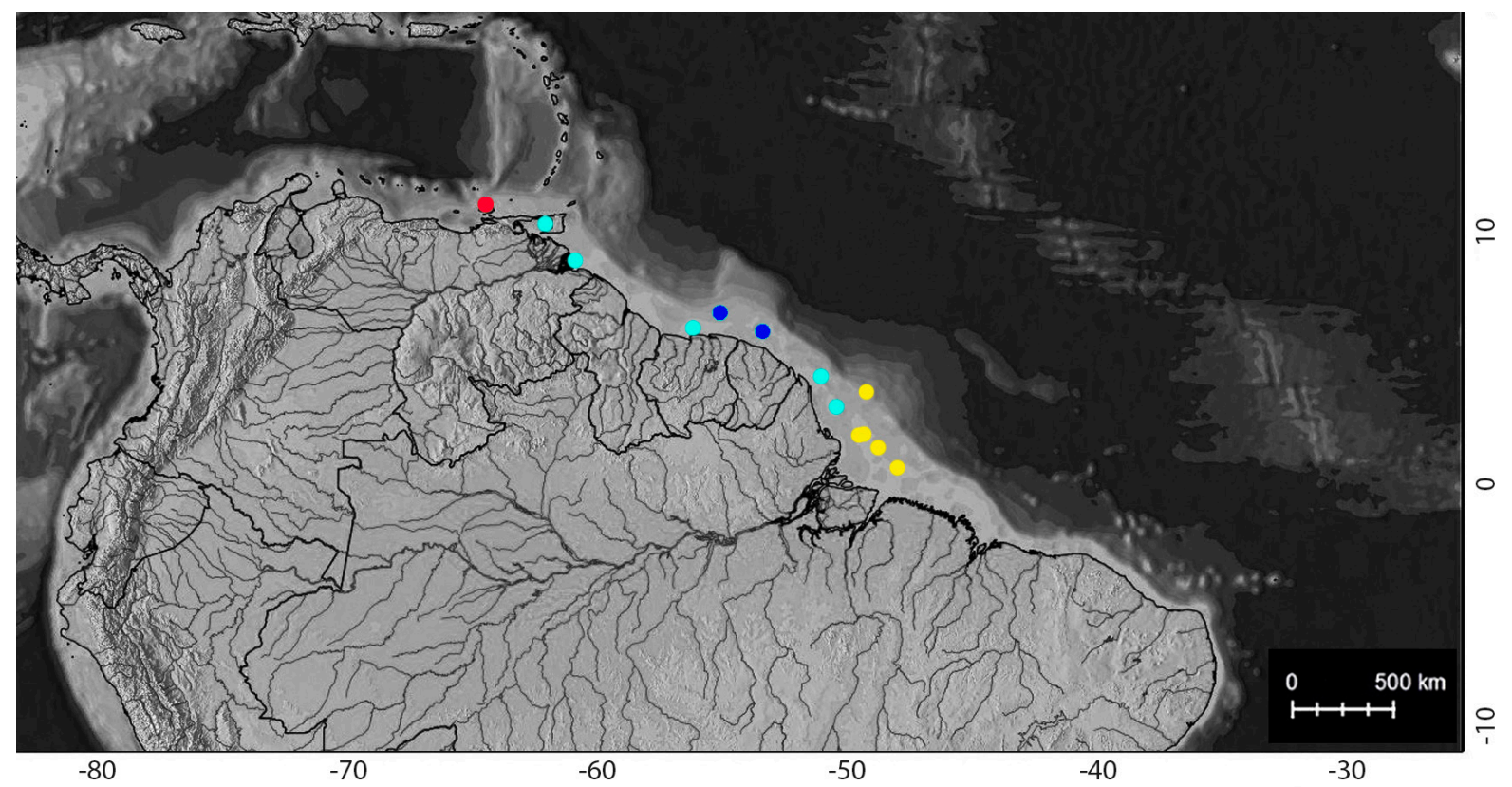

Figure 5. Map of the Atlantic coast of northern South America, showing the geographic distribution of Cynoscion similis records. Red: holotype; dark blue: paratypes; light blue: previous records in zoological collections; yellow: new records of the species from the northern Brazilian coast. This figure is in color in the electronic version. 
evidence from the other morphological parameters, as well as the DNA barcode, that this difference is derived from individual variation, or different ways to count gill rakers.

\section{DISCUSSION}

While $C$. similis had not previously been recorded from the northern Brazilian state of Pará, our examination of material obtained from CEPNOR indicated that the fish identified by Maia et al. (2016) as pescada-negra is, in fact, Cynoscion similis, given that this species is the only Atlantic sciaenid that presents dark pigmentation. In the same way, quite possibly the species went undetected in other inventory studies of local industrial fisheries, recorded as Cynoscion sp. (Pinheiro and Frédou 2004; Paiva et al. 2009).

Whereas Randall and Cervigón (1968) reported that $C$. similis and $C$. jamaicensis typically occurred in the same area, this was not so common on the northern Brazilian coast. Cynoscion similis showed to be common on the Brazilian continental shelf (at depths of 40-80 m), targeted by industrial trawling fisheries in Pará, while $C$. jamaicensis is more common in the coastal zone, at depths of less than $40 \mathrm{~m}$, where it is typically exploited by artisanal gillnet fisheries. Quite possibly Cynoscion similis is harvested in Brazil and marketed as goete or pescada gó, the local name for $C$. jamaicensis. This reinforces the need for reliable taxonomical identification of species, in order to guarantee the collection of accurate data on their ecology and fisheries, and ultimately, support the development of effective conservation strategies.

\section{CONCLUSIONS}

Despite its importance in biogeographical, ecological, and commercial terms, the fish fauna of the northern Brazilian coast is still poorly known, and this region is the least well sampled of the Brazilian Exclusive Economic Zone (Marceniuk et al. 2013). Our results indicate that $C$. similis is common throughout the northern coast of Brazil. The study also provides new morphological and molecular data to distinguish C. similis more reliably from the closely allied $C$. jamaicensis.

\section{ACKNOWLEDGMENTS}

This study was supported by the Conselho Nacional de Desenvolvimento Científico e Tecnológico (CNPq grants \# 300462/2016-6 to APM and \# 306054/2006-0 to CO), the Fundação Amazônia de Amparo a Estudos e Pesquisa do Pará (FAPESPA grant ICAAF \# 017/2016 to APM), and the Fundação de Amparo à Pesquisa do Estado de São Paulo (FAPESP grant \# 2014/26508-3, 2016/09204-6 to CO). The authors are grateful to Alex Garcia Cavalleiro de Macedo Klautau, coordinator of the Centro de Pesquisa e Gestão de Recursos Pesqueiros do Litoral Norte (CEPNOR), and the project Areas Marinhas e Costeiras Protegidas - GEF Mar of the Brazilian Federal Government, responsible for all material examined.

\section{REFERENCES}

Aguillera, O. 1998. Los peces marinos del occidente de Venezuela. Acta Biologica Venezuelica, 18: 43-57.

Castro-Aguirre, J.L.; Espinosa-Perez, H.; Schmitter-Soto, J.J. 1999. Ictiofauna estuarino-lagunar y vicaria de México. Colección Textos Politécnicos, Editorial Limusa, México D.F. 711p.

Casatti, L.; Menezes, N.A. 2003. Família Sciaenidae. In: Menezes, N.A.; Buckup, P.A; Figueiredo, J.L; Moura, R.L. (Ed.). Catálogo das espécies de peixes marinhos do Brasil. Museu de Zoologia da Universidade de São Paulo, São Paulo, p.86-89.

Cervigón, F. 1992. Tiburones, peces batoideos y peces óseos. In: Cervigón, F.; Cipriani, R.; Fischer, W.; Garibaldi, L.; Hendrickx, M.; Lemus, A.J.; Márquez, R.; Poutiers, J.M.; Robaina, G.; Rodríguez, B. (Ed.). Guía de campo de las especies comerciales marinas y de aguas salobres de la costa septentrional de Sur América. Fichas FAO de identificatión de especies para los fines de la pesca, FAO, Rome, p.163-456.

Cervigón, F. 1993. Los peces marinos de Venezuela. Fundación Científica Los Roques, Caracas, 499p.

Chao, L.N. 1978. A basis for classifying western Atlantic Sciaenidae (Teleostei: Perciformes). NOAA Technical Report NMFS (National Marine Fisheries Service), v. 415. U.S. Department of Commerce, National Marine Fisheries Service, 64p.

Chao, L.N. 2002. Taxonomy of the seatrout, genus Cynoscion (Pisces, Sciaenidae), with artificial keys to the species. In: Bortone, S.A. (Ed.). Biology of the spotted seatrout. CRC Marine Biology Series, London, p.5-15.

Chao, L.N. 2003. Sciaenidae. In: Carpenter, K.E. (Ed.). The living marine resources of the Western Central Atlantic. v.3. Bony fishes, part 2 (Opistognathidae to Molidae). FAO species identification guide for fishery purposes and American Society of Ichthyologist and Herpetologists Special Publication No. 5. FAO, Rome, p.1583-1653.

Chao, L.; Aquillera, O. 2015. Cynoscion similis. The IUCN Red List of Threatened Species 2015: e.T47147582A49238881. (http://dx.doi.org/10.2305/IUCN.UK.20152.RLTS. T47147582A49238881.en). Accessed on 06 May 2019.

Drummond, A.J.; Suchard, M.A.; Xie, D.; Rambaut, A. 2012. Bayesian phylogenetics with BEAUti and the BEAST 1.7. Molecular Biology and Evolution, 29: 1969-1973.

Edgar, R.C. 2004. Muscle: a multiple sequence alignment method with reduced time and space complexity. BMC Bioinformatics, 5: 113.

Kearse, M.; Moir, R.; Wilson, A.; Stones-Havas, S.; Cheung, M.; Sturrock, S. et al. 2012. Geneious Basic: An integrated and extendable desktop software platform for the organization and analysis of sequence data. Bioinformatics, 28: 1647-1649.

Lasso, C.A.; Lasso-Alcalá, O.M.; Capelo, J.C. 1999. Catálogo de la colección de tipos de peces de la fundación La Salle de ciencias naturales. Parte II: Museo Oceanológico Hermano Benigno Román (Mobr - edimar). Memoria de la Sociedad de Ciencias Naturales La Salle, 68: 105-114.

Maia, B.P.; Nunes, Z.M.P.; Holanda, F.C.A.F.; Silva, V.H.S.; Silva, B.B. 2016. Gradiente latitudinal da beta diversidade da fauna acompanhante das pescarias industriais de camaróes marinhos da costa Norte do Brasil. Biota Amazonica, 6: 31-39. 
Marceniuk, A.P.; Caires, R.A.; Wosiacki, W.B.; Di Dario, F. 2013. Conhecimento e conservação dos peixes marinhos e estuarinos (Chondrichthyes e Teleostei) da Costa Norte do Brasil. Biota Neotropica, 13: 251-259.

McEachran, J.D.; Fechhelm, J.D. 2005. Fishes of the Gulf of Mexico. v.2. University of Texas Press, Austin, 1004p.

Miller, M.A.; Pfeiffer, W.; Schwartz, T. 2010. Creating the CIPRES Science Gateway for inference of large phylogenetic trees. Proceedings of the Gateway Computing Environments Workshop (GCE), New Orleans, p.1-8.

Paiva, K.S.; Aragão, J.A.N.; Silva, K.C.A.; Cintra, I.H.A. 2009. A fauna acompanhante da pesca industrial do camarão - rosa na plataforma continental norte brasileira. Boletim Técnico Científico Cepnor, 9: 25-42.

Pinheiro, L.A.; Frédou, F.L. 2004. Caracterização geral da pesca industrial desembarcada no estado do Pará. Revista virtual de Iniciação Científica da Universidade Federal do Pará. (http://www. ufpa.br/revistaic). Accessed on 09 May 2019.

Randall, J.E.; Cervigón, F. 1968. Un nuevo pez Sciaenidae del genero Cynoscion de Venezuela y notas sobre $C$. jamaicensis y C. obliquatus. Contribución No. 28, Estación de Investigaciones Marinas de Margarita, Fundación La Salle de Ciencias Naturales. Memoria de la Sociedad de Ciencias Naturales La Salle, 27: 176-192.
Santos, S.R.; Vianna, M. 2017. Scientometric analysis of the fisheries science for the species of Cynoscion (Sciaenidae: Perciformes) from the Western Atlantic, with emphasis in the comparison of the North American and Brazilian fisheries catch data. Reviews in Fisheries Science \& Aquaculture, 26: 1-15.

Tamura, K.; Stecher, G.; Peterson, D.; Filipski, A.; Kumar, S. 2013. MEGA6: molecular evolutionary genetics analysis, version 6.0. Molecular Biology and Evolution, 30: 2725-2729.

Stamatakis, A.; Hoover, P.; Rougemont, J. 2008. A rapid bootstrap algorithm for the raxml web servers. Systematic Biology, 57: 758-771.

Uyeno, T.; Matsuura, K.; Fujii, E. 1983. Fishes trawled off Suriname and French Guiana. Japan Marine Fishery Resource Research Center, Tokyo, 520p.

Ward, R.D.; Zemlak, T.S.; Innes, B.H.; Last, P.R.; Hebert, P.D.N. 2005. DNA barcoding Australia’s fish species. Philosophical Transactions of the Royal Society B: Biological Sciences, 360: 1847-1857.

RECEIVED: 01/09/2018

ACCEPTED: 03/05/2019

ASSOCIATE EDITOR: Helder Mateus Espirito-Santo

\section{SUPPLEMENTARY MATERIAL}

(only available in the electronic version)

CAIRES et al. The Tonkin weakfish, Cynoscion similis (Sciaenidae, Perciformes), an endemic species of the Amazonas-Orinoco Plume

Appendix S1. Nucleotide differences observed in COI sequences among the samples analyzed. Numbers represent relative position in relation to the COI sequence of Cynoscion nebulosus (Genbank number JN021296).

\begin{tabular}{|c|c|c|c|c|c|c|c|c|c|c|c|c|c|c|c|c|c|c|c|c|}
\hline JN021296 & 9 & 10 & 12 & 15 & 18 & 24 & 30 & 33 & 36 & 45 & 45 & 48 & 54 & 57 & 60 & 63 & 66 & 69 & 70 & 72 \\
\hline Cynoscion acoupa & A & $G$ & $\mathrm{~T}$ & $\mathrm{~T}$ & $\mathrm{~T}$ & $A$ & $A$ & $G$ & $\mathrm{~T}$ & $\mathrm{~T}$ & $A$ & C & A & C & A & G & A & C & A & $\mathrm{T}$ \\
\hline Cynoscion arenarius & . & . & . & C & . & G & . & $A$ & . & . & . & $\mathrm{T}$ & . & . & . & A & . & . & . & . \\
\hline Cynoscion guatucupa & . & A & . & . & . & G & . & . & . & . & . & $\mathrm{T}$ & . & . & . & $A$ & G & $\mathrm{T}$ & . & . \\
\hline Cynoscion jamaicensis & - & - & - & - & - & - & - & - & - & - & - & - & . & . & . & A & . & . & . & C \\
\hline Cynoscion leiarchus & . & . & . & . & . & . & . & A & C & C & G & . & G & $\mathrm{T}$ & . & A & . & . & . & . \\
\hline Cynoscion microlepidotus & . & . & . & . & . & . & . & . & . & . & G & $\mathrm{T}$ & G & $\mathrm{T}$ & . & A & . & . & $C$ & . \\
\hline Cynoscion nothus & . & . & . & C & . & . & G & $A$ & . & . & . & $\mathrm{T}$ & . & . & . & A & . & . & . & . \\
\hline Cynoscion regalis & $\mathrm{G}$ & $A$ & . & C & . & G & . & $A$ & . & . & . & . & . & . & . & A & . & . & . & . \\
\hline Cynoscion similis & . & . & . & C & . & G & G & $A$ & . & . & . & $\mathrm{T}$ & . & . & $\mathrm{T}$ & A & . & . & . & C \\
\hline Cynoscion virescens & - & - & - & - & - & - & - & - & - & - & - & - & - & - & - & - & - & - & - & - \\
\hline JN021296 & 75 & 78 & 81 & 84 & 85 & 87 & 93 & 96 & 99 & 102 & 105 & 106 & 108 & 111 & 114 & 117 & 120 & 126 & 129 & 132 \\
\hline Cynoscion acoupa & A & $A$ & $\mathrm{~T}$ & $G$ & A & $\mathrm{T}$ & $A$ & $\mathrm{~T}$ & $C$ & A & G & C & $\mathrm{T}$ & $C$ & $\mathrm{~T}$ & $\mathrm{~T}$ & $\mathrm{~T}$ & $A$ & $A$ & C \\
\hline Cynoscion guatucupa & G & C & C & . & $\mathrm{G}$ & . & . & . & . & G & . & . & . & . & A & . & . & . & C & . \\
\hline Cynoscion jamaicensis & . & . & C & . & . & . & . & . & . & . & . & . & . & . & A & . & . & . & . & . \\
\hline Cynoscion leiarchus & . & . & C & . & . & . & . & . & $\mathrm{T}$ & G & . & . & . & . & A & C & . & . & . & . \\
\hline Cynoscion microlepidotus & . & . & C & . & . & $C$ & . & $C$ & . & G & . & $\mathrm{T}$ & . & . & $A$ & C & . & . & . & . \\
\hline Cynoscion nebulosus & . & . & C & . & . & . & . & . & . & . & $A$ & . & . & . & A & . & . & . & . & $\mathrm{T}$ \\
\hline Cynoscion nothus & . & . & C & . & . & . & . & . & . & G & . & . & . & . & $A$ & . & . & . & . & . \\
\hline Cynoscion regalis & . & . & C & . & C & . & . & . & . & G & . & . & . & . & A & . & . & G & G & . \\
\hline Cynoscion similis & . & . & $C$ & . & . & . & . & . & . & . & . & . & . & . & A & . & . & . & . & . \\
\hline Cynoscion virescens & - & - & C & . & . & . & . & & $\mathrm{T}$ & G & . & . & C & $\mathrm{T}$ & A & . & . & G & . & \\
\hline
\end{tabular}


ACTA

AMAZONICA

CAIRES et al. An endemic species of the Amazonas-Orinoco Plume

Appendix S1. Continued.

\begin{tabular}{|c|c|c|c|c|c|c|c|c|c|c|c|c|c|c|c|c|c|c|c|c|}
\hline JN021296 & 138 & 144 & 162 & 165 & 171 & 174 & 177 & 180 & 183 & 186 & 196 & 196 & 198 & 201 & 202 & 210 & 213 & 216 & 219 & 225 \\
\hline Cynoscion acoupa & C & A & A & C & A & $T$ & A & G & $C$ & G & A & G & G & A & C & $\mathrm{T}$ & A & C & $C$ & A \\
\hline Cynoscion arenarius & 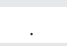 & & G & & G & & . & & $\mathrm{T}$ & 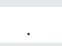 & $\mathrm{T}$ & & A & 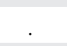 & & C & G & . & $\mathrm{T}$ & . \\
\hline Cynoscion guatucupa & & G & G & . & & . & & . & . & A & & A & C & & . & & & & & . \\
\hline Cynoscion jamaicensis & & & G & & & C & & . & . & & T & A & C & C & & C & G & & & G \\
\hline Cynoscion leiarchus & & . & G & . & & & & . & T & A & C & A & C & C & . & & & & & G \\
\hline Cynoscion microlepidotus & $\mathrm{T}$ & & G & & G & C & $r^{\prime}$ & . & $\mathrm{T}$ & A & $\mathrm{T}$ & A & C & G & & C & & 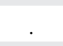 & & G \\
\hline Cynoscion nebulosus & . & . & & . & G & . & . & . & . & A & T & A & $\mathrm{T}$ & . & . & C & G & . & T & . \\
\hline Cynoscion nothus & $\mathrm{T}$ & & & . & & & & & & A & $\mathrm{T}$ & & A & & $\mathrm{T}$ & & & & & \\
\hline Cynoscion regalis & . & . & G & . & . & . & G & $\mathrm{T}$ & T & A & T & & . & & . & . & G & & $\mathrm{T}$ & . \\
\hline Cynoscion similis & $\mathrm{T}$ & . & . & . & G & C & . & & & A & $\mathrm{T}$ & & $A$ & . & & . & & & . & G \\
\hline Cynoscion virescens & & . & G & T & & . & . & . & . & & $\mathrm{T}$ & & A & 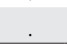 & . & . & G & $\mathrm{T}$ & & G \\
\hline JN021296 & 231 & 234 & 237 & 240 & 243 & 246 & 249 & 252 & 255 & 258 & 261 & 262 & 264 & 267 & 270 & 273 & 276 & 279 & 280 & 282 \\
\hline Cynoscion acoupa & $C$ & $\mathrm{~T}$ & A & G & $T$ & $T$ & G & C & C & A & $\bar{T}$ & C & A & C & $T$ & $T$ & C & C & C & 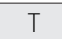 \\
\hline Cynoscion arenarius & & & & A & & & & . & . & & . & & C & & & . & . & & & A \\
\hline Cynoscion guatucupa & & C & & A & C & C & & . & T & G & C & T & G & & & . & . & & $\mathrm{T}$ & G \\
\hline Cynoscion jamaicensis & & & & & & & & . & . & . & . & & C & & & . & . & $\mathrm{T}$ & & G \\
\hline Cynoscion leiarchus & & 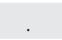 & C & . & C & C & & T & . & & & & C & A & C & & 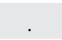 & T & & G \\
\hline Cynoscion microlepidotus & & C & & A & & & & . & . & . & & & & A & C & C & . & & & A \\
\hline Cynoscion nebulosus & & C & C & . & $C$ & . & & . & . & . & C & & T & & . & C & . & & & A \\
\hline Cynoscion nothus & $\mathrm{T}$ & . & & & C & & A & & & & & . & $\mathrm{T}$ & . & C & C & . & . & . & . \\
\hline Cynoscion regalis & & . & & A & & . & & . & . & G & . & & C & & . & . & . & & & A \\
\hline Cynoscion similis & . & & 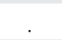 & A & & & & & & . & . & & C & T & & . & $\mathrm{T}$ & & & $A$ \\
\hline Cynoscion virescens & & . & & A & C & . & & . & . & & G & & $\mathrm{T}$ & & C & & . & & & G \\
\hline JN021296 & 285 & 286 & 288 & 291 & 294 & 297 & 300 & 303 & 306 & 309 & 312 & 315 & 318 & 321 & 324 & 327 & 330 & 333 & 336 & 339 \\
\hline Cynoscion acoupa & C & $C$ & A & C & $T$ & A & G & A & A & A & A & $C$ & G & A & A & G & A & $C$ & $T$ & $C$ \\
\hline Cynoscion arenarius & & & & & & . & & . & G & & G & & & & & A & . & & C & . \\
\hline Cynoscion guatucupa & $\mathrm{T}$ & . & & . & & . & & . & . & G & & & A & C & C & A & . & G & & . \\
\hline Cynoscion jamaicensis & & & & & & . & & . & . & & G & & A & & C & A & . & & C & . \\
\hline Cynoscion leiarchus & $\mathrm{T}$ & . & & . & & . & & $\mathrm{T}$ & . & G & G & & A & & G & A & . & T & C & . \\
\hline Cynoscion microlepidotus & . & T & . & & A & & . & & . & . & G & & A & G & C & A & . & $\mathrm{T}$ & & . \\
\hline Cynoscion nebulosus & . & . & . & . & . & . & A & . & G & . & G & & . & . & G & A & . & T & C & . \\
\hline Cynoscion nothus & & & & & & & A & & G & & G & & . & & C & A & & & C & \\
\hline Cynoscion regalis & & . & & T & & . & & . & G & G & . & & . & G & G & & . & & C & 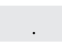 \\
\hline Cynoscion similis & & & G & & & & C & 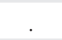 & G & G & . & & & & C & A & . & T & C & . \\
\hline Cynoscion virescens & & & $\mathrm{T}$ & 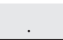 & & . & A & & G & G & . & $\mathrm{T}$ & A & & . & & & G & C & \\
\hline JN021296 & 342 & 345 & 348 & 351 & 354 & 357 & 360 & 363 & 366 & 369 & 372 & 375 & 378 & 381 & 382 & 384 & 387 & 390 & 393 & 397 \\
\hline Cynoscion acoupa & $A$ & C & A & $T$ & $C$ & $C$ & A & C & A & A & $C$ & $C$ & $C$ & $\mathrm{~T}$ & $T$ & A & C & $C$ & $C$ & $C$ \\
\hline Cynoscion arenarius & & & G & G & & G & & . & . & G & . & & & & & . & . & $\mathrm{T}$ & & $\mathrm{T}$ \\
\hline Cynoscion guatucupa & $\mathrm{T}$ & . & T & C & & . & & T & G & C & . & $\mathrm{T}$ & . & C & C & G & . & . & $\mathrm{T}$ & . \\
\hline Cynoscion jamaicensis & . & & G & C & . & $\mathrm{T}$ & C & & & G & & . & & C & & . & . & . & . & . \\
\hline Cynoscion leiarchus & . & T & & A & . & $\mathrm{T}$ & . & . & . & G & $\mathrm{T}$ & & . & C & C & . & . & . & . & . \\
\hline Cynoscion microlepidotus & & T & G & C & T & A & & T & & G & T & & . & C & . & & & & & \\
\hline Cynoscion nebulosus & & . & & C & & $\mathrm{T}$ & & . & . & & T & $\mathrm{T}$ & $\mathrm{T}$ & C & C & & . & . & . & . \\
\hline Cynoscion nothus & G & T & & C & & & C & & & G & & & $\mathrm{T}$ & C & . & & T & & & \\
\hline Cynoscion regalis & & . & G & G & & $G$ & & . & . & G & . & & . & C & . & G & . & & & . \\
\hline Cynoscion similis & G & & G & C & 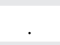 & $\mathrm{T}$ & C & & & G & . & & & C & & G & & . & . & \\
\hline Cynoscion virescens & G & T & G & 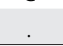 & & $\mathrm{T}$ & & T & & G & T & & . & C & . & G & & & T & \\
\hline JN021296 & 399 & 402 & 405 & 408 & 414 & 417 & 420 & 423 & 424 & 426 & 429 & 432 & 435 & 441 & 444 & 447 & 450 & 454 & 456 & 459 \\
\hline Cynoscion acoupa & $\mathrm{C}$ & C & $\mathrm{T}$ & $A$ & $C$ & $A$ & $A$ & $T$ & $C$ & $A$ & $G$ & $\mathrm{~T}$ & $\mathrm{~T}$ & $\mathrm{~T}$ & $\mathrm{~T}$ & A & $A$ & $C$ & $T$ & C \\
\hline Cynoscion arenarius & A & & C & & . & & . & & $\mathrm{T}$ & . & & C & & . & & . & & $\mathrm{T}$ & & . \\
\hline Cynoscion guatucupa & $\mathrm{T}$ & . & C & . & . & . & . & . & T & . & . & C & C & & . & C & C & $\mathrm{T}$ & & . \\
\hline Cynoscion jamaicensis & & & C & . & & & & & & & & & . & & & & & $\mathrm{T}$ & & $\mathrm{T}$ \\
\hline Cynoscion leiarchus & T & $\mathrm{T}$ & & T & T & . & & C & . & $\mathrm{T}$ & . & & C & & . & & . & T & & $\mathrm{T}$ \\
\hline Cynoscion microlepidotus & G & T & C & . & G & C & G & & & & & & C & C & . & & C & $\mathrm{T}$ & & $\mathrm{T}$ \\
\hline Cynoscion nebulosus & & . & C & . & . & . & . & . & T & . & . & . & . & . & . & . & . & $\mathrm{T}$ & & $\mathrm{T}$ \\
\hline Cynoscion nothus & A & & C & & 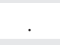 & & & & & & & C & & 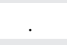 & & . & & $\mathrm{T}$ & & $\mathrm{T}$ \\
\hline Cynoscion regalis & A & . & C & . & & & G & & & G & & C & . & & . & & . & T & & . \\
\hline Cynoscion similis & & & & & . & & $\mathrm{T}$ & & & & & C & & & C & & . & $\mathrm{T}$ & & T \\
\hline Cynoscion virescens & & 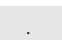 & & & $\mathrm{T}$ & . & & & . & & & & & & . & & C & $\mathrm{T}$ & & $\mathrm{T}$ \\
\hline
\end{tabular}


ACTA

AMAZONICA

CAIRES et al. An endemic species of the Amazonas-Orinoco Plume

Appendix S1. Continued.

\begin{tabular}{|c|c|c|c|c|c|c|c|c|c|c|c|c|c|c|c|c|c|c|c|c|}
\hline JN021296 & 462 & 465 & 468 & 471 & 474 & 477 & 480 & 483 & 486 & 489 & 492 & 495 & 496 & 498 & 504 & 507 & 510 & 513 & 514 & 525 \\
\hline Cynoscion acoupa & $G$ & A & $T$ & $T$ & $T$ & $T$ & $C$ & $G$ & $T$ & G & $T$ & C & $T$ & A & A & A & $C$ & $T$ & $C$ & $T$ \\
\hline Cynoscion arenarius & & & C & & & . & $\mathrm{T}$ & A & & A & A & & & . & G & . & G & . & & \\
\hline Cynoscion guatucupa & . & . & C & . & $C$ & C & $\mathrm{T}$ & . & . & A & G & $\mathrm{T}$ & C & G & G & . & . & . & $\mathrm{T}$ & C \\
\hline Cynoscion jamaicensis & & & C & . & & C & & & & & A & & . & & . & G & $\mathrm{T}$ & C & & \\
\hline Cynoscion leiarchus & A & . & . & G & 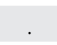 & . & . & . & C & A & A & T & . & 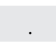 & $\mathrm{T}$ & G & . & . & 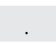 & $C$ \\
\hline Cynoscion microlepidotus & & . & . & $C$ & 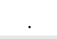 & C & . & & & & A & $\mathrm{T}$ & C & G & & G & & $C$ & & \\
\hline Cynoscion nebulosus & A & . & $C$ & . & & . & & A & . & & A & & C & $C$ & . & & & & & C \\
\hline Cynoscion nothus & & & C & & & & & A & . & A & G & & & & & G & $\mathrm{T}$ & & & . \\
\hline Cynoscion regalis & A & . & $C$ & . & 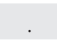 & . & . & A & . & 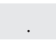 & A & & . & & G & G & A & & & . \\
\hline Cynoscion similis & & & . & $C$ & . & C & . & & & & $A$ & 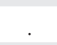 & C & . & G & G & & $C$ & $\mathrm{~T}$ & C \\
\hline Cynoscion virescens & & G & C & C & & C & & - & . & & A & $\mathrm{T}$ & C & & G & & 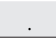 & C & & C \\
\hline JN021296 & 528 & 531 & 532 & 534 & 537 & 540 & 543 & 546 & 549 & 552 & 553 & 555 & 558 & 564 & 567 & 570 & 573 & 576 & 577 & 579 \\
\hline Cynoscion acoupa & $\mathrm{T}$ & C & $C$ & G & A & $C$ & $C$ & $C$ & $T$ & $C$ & $T$ & A & $\mathrm{T}$ & $C$ & $C$ & A & $G$ & $\mathrm{~T}$ & C & $\mathrm{T}$ \\
\hline Cynoscion arenarius & & & & . & & & G & & & & & & . & & $\mathrm{T}$ & $\mathrm{T}$ & & & & A \\
\hline Cynoscion guatucupa & $C$ & $\mathrm{~T}$ & & A & & T & A & $\mathrm{T}$ & . & . & . & G & C & & $\mathrm{T}$ & . & . & . & . & A \\
\hline Cynoscion jamaicensis & $C$ & & . & & - & - & - & - & - & - & - & - & - & - & - & - & - & - & - & - \\
\hline Cynoscion leiarchus & $C$ & G & & A & & $\mathrm{T}$ & A & G & . & $\mathrm{T}$ & . & & . & & $\mathrm{T}$ & & 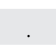 & & & C \\
\hline Cynoscion microlepidotus & $C$ & & $\mathrm{~T}$ & A & G & & G & . & . & 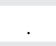 & . & 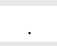 & & $\mathrm{T}$ & $\mathrm{T}$ & . & A & C & & . \\
\hline Cynoscion nebulosus & & . & & & T & . & A & . & C & A & . & & . & & $\mathrm{T}$ & & & & & A \\
\hline Cynoscion nothus & & & & $A$ & & . & A & & C & & . & . & . & . & $\mathrm{T}$ & & & & . & G \\
\hline Cynoscion regalis & . & . & . & . & & . & A & . & C & . & . & . & . & . & $\mathrm{T}$ & . & A & . & 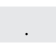 & A \\
\hline Cynoscion similis & & & & . & & & A & $\mathrm{T}$ & C & & & & . & & . & & & & & A \\
\hline Cynoscion virescens & . & . & 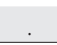 & A & & G & G & - & - & - & - & - & - & - & - & - & - & - & - & - \\
\hline JN021296 & 582 & 585 & 588 & 591 & 592 & 594 & 597 & 600 & 6044 & 609 & 615 & 618 & 621 & 622 & 625 & 630 & 633 & 639 & 642 & 645 \\
\hline Cynoscion acoupa & A & C & $C$ & $T$ & $C$ & G & $T$ & A & C & $C$ & A & A & A & A & A & C & $T$ & $T$ & $T$ & A \\
\hline Cynoscion arenarius & 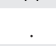 & . & . & & $\mathrm{T}$ & A & & . & . & . & G & & G & & & 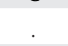 & . & & & . \\
\hline Cynoscion guatucupa & & . & & $C$ & & A & & . & . & . & . & & . & G & $\mathrm{T}$ & $\mathrm{T}$ & A & & & . \\
\hline Cynoscion jamaicensis & - & - & - & - & - & - & - & - & - & - & - & - & - & - & - & - & - & - & - & - \\
\hline Cynoscion leiarchus & $C$ & $\mathrm{~T}$ & G & $C$ & & A & & & . & $\mathrm{T}$ & $\mathrm{T}$ & & . & $\mathrm{T}$ & G & $\mathrm{T}$ & & & & . \\
\hline Cynoscion microlepidotus & & & & $C$ & & C & C & & . & & $C$ & & & G & G & & A & C & & . \\
\hline Cynoscion nebulosus & & . & & & $\mathrm{T}$ & A & & & $\mathrm{T}$ & & G & & . & & G & $\mathrm{T}$ & & & C & \\
\hline Cynoscion nothus & . & & . & $C$ & . & A & . & & & $\mathrm{T}$ & & . & G & . & G & . & C & . & C & . \\
\hline Cynoscion regalis & . & . & & . & $\mathrm{T}$ & A & & . & . & & . & & G & & G & $\mathrm{T}$ & . & & C & \\
\hline Cynoscion similis & r. & & . & & . & $\mathrm{T}$ & . & & & $\mathrm{T}$ & G & & G & . & & $\mathrm{T}$ & . & . & C & . \\
\hline Cynoscion virescens & - & - & - & - & - & - & - & - & - & - & - & - & - & - & - & - & - & - & - & - \\
\hline
\end{tabular}

This is an Open Access article distributed under the terms of the Creative Commons Attribution License, which permits unrestricted use, distribution, and reproduction in any medium, provided the original work is properly cited. 